\title{
POSTANTIBIOTIC EFFECTS AND POSTANTIBIOTIC SUB-MIC EFFECTS OF TILMICOSIN, ERYTHROMYCIN AND TIAMULIN ON ERYTHROMYCIN-RESISTANT STREPTOCOCCUS SUIS
}

\author{
Liping Wang *; Yuanshu Zhang
}

College of Veterinary Medicine, Nanjing Agricultural University, Nanjing 210095, China

Submitted: October 20, 2008; Returned to authors for corrections: December 01, 2008; Approved: May 15, 2009.

\begin{abstract}
The postantibiotic effects (PAEs) and postantibiotic sub-MIC effects (PA SMEs) of tilmicosin, erythromycin and tiamulin on erythromycin-susceptible and erythromycin-resistant strains of Streptococcus suis (M phenotype) were investigated in vitro. Tilmicosin and tiamulin induced significantly longer PAE and PA SME against both erythromycin-susceptible and erythromycin-resistant strains than did erythromycin. The durations of PAE and PA SMEs were proportional to the concentrations of drugs used for exposure. The PA SMEs were substantially longer than PAEs on S. suis $(P<0.05)$ regardless of the antimicrobial used for exposure. The results indicated that the PAE and PA SME could help in the design of efficient control strategies for infection especially caused by erythromycin-resistant S. suis and that they may provide additional valuable information for the rational drug use in clinical practice.
\end{abstract}

Key words: Postantibiotic effects, postantibiotic sub-MIC effects, tilmicosin, tiamulin, erythromycin, erythromycin-resistant Streptococcus suis

\section{INTRODUCTION}

Streptococcus suis is one of the principal etiologic agents of contagious bacterial disease in pigs. It can cause a variety of clinical syndromes including meningitis, arthritis, pericarditis, polyserositis, septicaemia, pneumonia. Infection may cause death in weaning piglets as well as growing pigs. The disease has a worldwide distribution and causes considerable losses to pig production (12). As an emerging zoonotic pathogen, S. suis can be transmitted to human beings by direct contact $(16,18,35)$. In 2005, an unprecedented outbreak in China resulted in more than 200 human cases that were directly linked to a concurrent outbreak of S. suis infection in pigs. About $20 \%$ of these human cases were fetal $(18,35)$.

At present, prevention and control of the disease are based on the use of autogenous vaccine and antimicrobial drugs (32). Macrolides and fluroquinolone, such as erythromycin, tylosin and enrofloxacin, have been considered as the effective substitutes for penicillin because $S$. suis isolates are historically highly susceptible to these drugs. However, some of the commonly used antibiotics (erythromycin, enrofloxacin, etc) for treatment and prevention of $S$. suis infections in pigs are becoming less effective, due to an increase in resistance among $S$. suis isolates in recent years $(11,30,32,36)$. Now more active 
FQs and new macrolides are becoming available, but new treatment strategies must accompany use of those agents to halt the selection of resistant mutants before the entire quinolone and macrolide class of drugs becomes ineffective.

The postantibiotic effect (PAE) is defined as the suppression of bacterial growth persisting after a short exposure of bacterial cultures to an antibiotic. The effect of sub-MIC concentration on growth during the PAE period has been defined as the post-antibiotic subinhibitory effect (PA SMEs). The two effects are one of the factors that have a clinical impact on antibacterial dosing regimens, allowing less frequent dosing than with agents with no PAE (7). They are now a well-established feature of virtually all antimicrobial agents and have been observed for a wide variety of bacterial and yeasts $(8,25,26)$.

To our knowledge, no data are available on the PAE of macrolides against $S$. suis, especially PAEs of 16-membered ring macrolides on erythromycin-resistant $S$. suis. Therefore, the purpose of the present work was to evaluate in vitro the PAEs and PA SMEs of erythromycin, tilmicosin and tiamulin on erythromycin-susceptible and erythromycin-resistant $S$. suis strains with $\mathrm{M}$ phenotype.

\section{MATERIALS AND METHODS}

\section{Bacterial strains}

Fifteen strains of $S$. suis were isolated in 2007 from the liver and spleen of diseased weaning piglets in two swine farms from Jiangsu and Shanghai in China. In these two herds, usual antibiotics including macrolide, $\beta$-lactams, fluroquinolones are used to treat some infections caused by E. coli, Salmonalla, S. suis etc. SS2D strain was isolated from diseased pigs in Germany. The serotypes were determined as type 2 using a PCR method for detecting MRP and, EF virulence factors in $S$. suis $(21,34)$. In addition to the clinical S. suis isolates, three American type Culture Collection (ATCC) strains, as recommended by the Clinical and Laboratory Standards Institute (CLSI, formerly known as NCCLS) were included for quality control in the susceptibility testing. The quality control strains were $S$. pneumoniae ATCC 25922, S. aureus ATCC29213, and E. faecalis ATCC29212. All strains were kept at -70 until use. Prior to testings, All Streptococcus suis isolates were subcultured on Columbia blood agar plates with 5\% sheep blood and incubated overnight at 35 in an atmosphere containing $5 \% \mathrm{CO} 2$.

\section{Antibiotics}

Tilmicosin, tiamulin and erythromycin were obtained from China Institute of Veterinary Drug Control (IVDC, China). The disks of erythromycin and clindamycin were bought from Tianhe Corporation, Hangzhou, P.R., China.

\section{Susceptibility testing for erythromycin, tilmicosin and tiamulin}

The MICs were determined by a broth microdilution method using custom made, microtitre panels in MuellerHinton broth supplemented with $5 \%$ calf serum according to the CLSI (6). Serial 2-fold dilutions of the antibiotics were inoculated with overnight cultures of $S$. suis isolates at a final inoculum of $106 \mathrm{cfu} / \mathrm{mL}$. The two fold serial dilution ranges for the three antimicrobial agents were between $0.125 \sim 128 \mu \mathrm{g} / \mathrm{mL}$. One hundred microlitres of the final inoculum and antibiotics were dispensed into each well of the microtitre plate panel. The plates were incubated aerobically at $35^{\circ} \mathrm{C}$ for $1824 \mathrm{~h}$. The MIC for each isolate was determined as the lowest dilution showing no visible growth. MBC was determined by plating $0.010 \mathrm{~mL}$ from the wells showing no visible growth on agar plates and incubating for 18-24 h. MBC was considered as the concentration at which a $99.9 \%$ reduction in cfu occurred, when compared with the original inoculum. The three quality control strains were included in duplicate in each batch tested.

\section{Erythromycin-resistant phenotype}

All erythromycin-resistant strains were analyzed for the phenotypes by double disk method described for Streptococcus pyogenes (28). A sterile cotton swab was 
Wang, L. et al.

dipped into a bacterial suspension with a turbidity matching that of $0.5 \mathrm{McF}$ arland standard and then streaked onto $\mathrm{MH}$ agar supplemented with 5\% sheep blood. The erythromycin $(15 \mu \mathrm{g})$ and clindamycin $(2 \mu \mathrm{g})$ disks were placed 15 to $20 \mathrm{~mm}$ apart on the agar surface. The plate was incubated overnight at $37^{\circ} \mathrm{C}$. A blunting of the clindamycin inhibition zone proximal to the erythromycin disk indicated inducible resistance (iMLS $\mathrm{B}_{\mathrm{B}}$ phenotype). The absence of an inhibition zone around the two disks was defined as constitutive resistance $\left(\mathrm{cMLS}_{\mathrm{B}}\right)$. Susceptibility to clindamycin with no blunting around the inhibition zone was interpreted as $\mathrm{M}$ phenotype.

For the detection of different erythromycin resistance genes in the genomes of $S$. suis by PCR, DNA was extracted using CASsuper Bacteria Genomic DNA Isolation Kit (Casarray Co., Shanghai, China). For the PCR amplification of ermB and mefA gene segments, primers ermB and mefA were used as reported by Sutcliffe (29). The expected amplicon sizes were $640 \mathrm{bp}$ for $\mathrm{ermB}, 348 \mathrm{bp}$ for $m e f A$. The isolates with mefA gene were $\mathrm{M}$ phenotype and those with ermB gene were constitutive-resistant (CR) and inducibleresistant (IR) phenotype. The nucleotide sequences of the amplification products from ermB and mefA positive $S$. suis isolates were determined and compared to published ermB and $m e f A$ gene sequences in NCBI GenBank database.

\section{Determination of PAE and PA SME}

To determine the PAE, bacterial cultures (approximately $10^{6} \mathrm{cfu} / \mathrm{ml}$ ) were first exposed to antibiotics at 1/4, 1/2,1 and 2 times the MIC for 1 and 2 hours, respectively. At the end of the exposed period, the antibiotic was removed by diluting $1: 10^{3}$ into the prewarmed, antibiotic free broth for three times. For quantitation of the PAE, viable counts were determined continuously before and after antibiotic exposure and then hourly for $24 \mathrm{~h}$ by plate counting method. PAE was expressed with the unit of hour and determined by the formula $\mathrm{PAE}=\mathrm{T}-\mathrm{C}$, where $\mathrm{T}$ was the time required for the antimicrobial exposed test culture to increase by $1 \log _{10}(10$ fold) after elimination of antibiotics, and $\mathrm{C}$ was the time required for the number of orgnisms in the control tube to increase by $1 \log (24)$.

To induce PA SMEs, bacterial cultures in postantibiotic phase (induced with 1/4 MIC for $2 \mathrm{~h}$ ) were exposed to antibiotics at 1/8, 1/4, 1/2 times the MIC. The same procedures described above for PAE were used thereafter. PASME was defined as follows: PASME=Tpa-C, where Tpa is the time for cultures previously exposed to antibiotic and then reexposed to different sub-MICs to increase by $1 \log _{10}$ above counts immediately after dilution and $\mathrm{C}$ is the cprresponding time for the unexposed control (23).

\section{Statistics}

Statistical analyses were performed using software SPSS 13.0. The differences between the duration of PAE of different drugs were analysed using two-tailed paired $t$-test, followed by Bonferroni's multiple comparison tests when appropriate. A $P$-value $<0.05$ was considered to be statistically significant.

\section{RESULTS}

\section{MICs and the phenotype of erythromycin-resistant $S$. suis}

The MICs and MBCs of tilmicosin, erythromycin and tiamulin for $S$. suis are given in Table1. Tilmicosin and tiamulin showed substantial activity against 16 strains with MICs being 0.03 0.13mg/L. Erythromycin was less active than tiamulin and tilmicosin having MICs between $0.03 \sim 32 \mathrm{mg} / \mathrm{L}$. The minimum bactericidal concentrations (MBC) of three antibiotics were 1 to 4 -fold higher than the MIC.

The results of double disk test and PCR method showed that strains SS-2B, SS2-H, HM, WHR1, WHR2, WHR3, WHR4 and SS2-G harbored mefA gene (described M phenotype), but other eight strains HA9801, SS2D, WHR, SS2-2, SS2-3, SS2-4, SS2-5 and Bei1 were susceptible to erythromycin, tilmicosin and tiamulin and negative for mefA and $\operatorname{erm} B$ gene. None strain was positive for ermB gene. All MIC values obtained with the quality control organisms were 
within the expected ranges. This confirmed that the test conditions were in agreement with the CLSI recommendations.

\section{Determination of PAEs}

The mean duration of the PAE $( \pm \mathrm{SD})$ in hours for erythromycin-susceptible and erythromycin-resistant strains to erythromycin, tilmicosin and tiamulin are displayed in Table 2 and Table 3, respectively. Each value represents three replicates. The results showed that the durations of PAE was directly related to the exposure time, it lasted longer when the strains were exposed to the antibiotics for two hours than those obtained after an hour exposure $(P<0.05)$. Increasing the concentrations of antimicrobials also prolonged the duration of PAEs, showing a linear relationship.

The durations of PAE of tiamulin and tilmicosin did not differ significantly between erythromycin-susceptible and erythromycin-resistant (with $\mathrm{M}$ phenotype) S. suis isolates $(P>0.05)$, confirming that $\mathrm{M}$ phenotype resistant strains were still susceptible to tiamulin and tilmicosin. Tiamulin induced a significantly longer PAE than tilmicosin at 1 time MIC and two times MIC at two hour exposure period $(P<0.05)$. Tiamulin exposure for one hour at two times MIC also resulted in longer PAE than did tilmicosin under the same condition $(P<0.05)$. However there were no significant differences in the activity of tilmicosin and tiamulin at concentrations of 1/4,1/2, 1 times MIC when exposed for one hour and at 1/4, 1/2 times MIC when exposed for 2 hours.

Increasing concentrations of erythromycin induced significantly longer PAEs against susceptible $S$. suis isolates than did the lower concentrations. However, that regrowth time of the resistant strains that had high MICs was not investigated in this study.

Table 1. MICs and phenotype of tilmicosin, erythromycin and tiamulin against S.suis type 2

\begin{tabular}{cccccc}
\hline \multirow{2}{*}{ Bacterial strains } & phenotype & mefA/ermB & \multicolumn{3}{c}{ MICs /MBCs (mg/L) } \\
\cline { 5 - 6 } & & & tilmicosin & tiamulin & erythromycin \\
\hline HA9801 & susceptible & $-/-$ & $0.13 / 0.25$ & $0.06 / 0.12$ & $0.13 / 0.5$ \\
SS2D & susceptible & $-/-$ & $0.06 / 0.12$ & $0.03 / 0.03$ & $0.06 / 0.25$ \\
WHR & susceptible & $-/-$ & $0.13 / 0.13$ & $0.06 / 0.06$ & $0.13 / 0.25$ \\
SS2-2 & susceptible & $-/-$ & $0.03 / 0.03$ & $0.03 / 0.06$ & $0.13 / 0.25$ \\
SS2-3 & susceptible & $-/-$ & $0.06 / 0.06$ & $0.13 / 0.25$ & $0.13 / 0.25$ \\
SS2-4 & susceptible & $-/-$ & $0.13 / 0.25$ & $0.03 / 0.06$ & $0.25 / 0.5$ \\
SS2-5 & susceptible & $-/-$ & $0.03 / 0.03$ & $<0.03 / 0.03$ & $0.13 / 0.25$ \\
Bei1 & susceptible & $-/-$ & $<0.03 / 0.03$ & $<0.03 / 0.03$ & $<0.03 / 0.03$ \\
SS2-H & M & $+/-$ & $0.13 / 0.25$ & $0.13 / 0.13$ & $32 / 64$ \\
SS2-B & M & $+/-$ & $0.03 / 0.03$ & $0.06 / 0.12$ & $>64 />64$ \\
HM & M & $+/-$ & $0.06 / 0.12$ & $0.13 / 0.25$ & $>64 />64$ \\
WHR1 & M & $+/-$ & $0.13 / 0.25$ & $0.13 / 0.25$ & $16 / 32$ \\
WHR2 & M & $+/-$ & $0.13 / 0.25$ & $0.06 / 0.12$ & $4 / 8$ \\
WHR3 & M & $+/-$ & $<0.03 / 0.03$ & $0.06 / 0.12$ & $8 / 8$ \\
WHR4 & M & $+/-$ & $<0.03 / 0.03$ & $0.13 / 0.25$ & $8 / 16$ \\
SS2-G & M & $+/-$ & $0.06 / 0.12$ & $0.06 / 0.12$ & $4 / 8$ \\
S. aureus ATCC29213 & - & - & $2 / 2$ & $0.5 / 0.5$ & $0.25 / 0.5$ \\
\hline faecalis ATCC29212 & - & - & $8 / 8$ & - & $1 / 1$ \\
\hline pneumoniae ATCC 25922 & & - & - & $1 /$ & $0.03 / 0.6$ \\
\hline
\end{tabular}


Wang, L. et al.

Table 2. PAEs (h) of erythromycin, tilmicosin and tiamulin against erythromycin-susceptiable strains of S.suis type 2. (n=8)

\begin{tabular}{ccccccccc}
\hline \multirow{2}{*}{ Agents } & \multicolumn{6}{c}{ PAE h, mean \pm SD at indicated fraction MIC } \\
\cline { 2 - 8 } & \multicolumn{3}{c}{ Exposure for 1h } & \multicolumn{4}{c}{ Exposure for 2h } \\
\cline { 2 - 8 } & $\mathbf{1 / 4 \times M I C}$ & $\mathbf{1 / 2 \times M I C}$ & $\mathbf{1} \times \mathbf{M I C}$ & $\mathbf{2 \times M I C}$ & $\mathbf{1 / 4 \times M I C}$ & $\mathbf{1 / 2 \times M I C}$ & $\mathbf{1 \times M I C}$ & $\mathbf{2 \times M I C}$ \\
\hline erythromycin & $0.53 \pm 0.17$ & $0.91 \pm 0.15$ & $1.79 \pm 0.17$ & $2.45 \pm 0.25$ & $1.28 \pm 0.12$ & $2.52 \pm 0.22$ & $3.49 \pm 0.28$ & $4.55 \pm 0.29$ \\
tilmicosin & $1.02 \pm 0.08$ & $1.89 \pm 0.11$ & $2.28 \pm 0.31$ & $3.03 \pm 0.08$ & $1.47 \pm 0.09$ & $2.76 \pm 0.15$ & $4.26 \pm 0.30$ & $5.75 \pm 0.09$ \\
tiamulin & $1.15 \pm 0.10$ & $2.02 \pm 0.15$ & $2.99 \pm 0.15$ & $3.59 \pm 0.53$ & $1.90 \pm 0.11$ & $2.90 \pm 0.17$ & $4.78 \pm 0.19$ & $6.11 \pm 0.39$ \\
\hline
\end{tabular}

a: PAE values (h) determined on 8 erythromycin-susceptiable S. suis. Data are means \pm SD.

Table 3. PAEs (h) of tilmicosin and tiamulin against erythromycin-resistant strains of S.suis type $2(\mathrm{n}=8)$

\begin{tabular}{ccccccccc}
\hline \multirow{2}{*}{ Agents } & \multicolumn{6}{c}{ PAE $\mathbf{~ h , ~ m e a n ~} \pm$ SD at indicated fraction MIC } \\
\cline { 2 - 8 } & \multicolumn{5}{c}{ Exposure for $\mathbf{1 h}$} \\
\cline { 2 - 8 } & $\mathbf{1 / 4 \times M I C}$ & $\mathbf{1 / 2} \times \mathbf{M I C}$ & $\mathbf{1} \times \mathbf{M I C}$ & $\mathbf{2 \times M I C}$ & $\mathbf{1 / 4 \times M I C}$ & $\mathbf{1 / 2 \times M I C}$ & $\mathbf{1 \times M I C}$ & $\mathbf{2 \times M I C}$ \\
\hline tilmicosin & $0.73 \pm 0.06$ & $1.52 \pm 0.19$ & $2.27 \pm 0.18$ & $2.76 \pm 0.11$ & $1.43 \pm 0.06$ & $2.46 \pm 0.09$ & $3.61 \pm 0.23$ & $5.6 \pm 0.09$ \\
tiamulin & $1.22 \pm 0.07$ & $1.88 \pm 0.3$ & $2.81 \pm 0.25$ & $3.74 \pm 0.31$ & $1.81 \pm 0.11$ & $2.71 \pm 0.17$ & $4.56 \pm 0.13$ & $6.05 \pm 0.24$ \\
\hline
\end{tabular}

a: PAE values (h) determined on 8 erythromycin-resistant S. suis. Data are means \pm SD.

\section{Determination of PA SMEs}

The results (Table 4) showed that the durations of PA SMEs were also proportional to the concentrations of drugs used for exposure. The PA SMEs of tiamulin and tilmicosin significantly longer than PAEs $(P<0.05)$; however, there were no significant differences between the PA SMEs of taimulin and tilmicosin $(P>0.05)$.

Table 4. PASMEs (h) of tilmicosin and tiamulin against erythromycin-susceptible and erythromycin-resistant strains of S.suis type 2

\section{PA SME $h$, mean \pm SD at indicated fraction MIC $^{\mathrm{a}}$}

\begin{tabular}{ccccccc} 
Agents & \multicolumn{2}{c}{ erythromycin-susceptiable $\boldsymbol{S}$. suis $(\mathbf{n}=\mathbf{8})$} & \multicolumn{3}{c}{ erythromycin-resistant $\boldsymbol{S}$. suis $(\mathbf{n}=\mathbf{8})$} \\
& $\mathbf{1 / 8} \times \mathbf{M I C}$ & $\mathbf{1 / 4 \times M I C}$ & $\mathbf{1 / 2 \times M I C}$ & $\mathbf{1 / 8} \times \mathbf{M I C}$ & $\mathbf{1 / 4 \times M I C}$ & $\mathbf{1 / 2 \times M I C}$ \\
\hline tilmicosin & $5.91 \pm 0.27$ & $7.49 \pm 0.30$ & $8.61 \pm 0.34$ & $6.08 \pm 0.25$ & $8.00 \pm 0.69$ & $8.92 \pm 0.35$ \\
tiamulin & $6.65 \pm 0.26$ & $8.18 \pm 0.21$ & $9.06 \pm 0.32$ & $6.86 \pm 0.33$ & $7.62 \pm 0.34$ & $8.56 \pm 0.42$ \\
\hline
\end{tabular}

a: PA SME values (h) determined on 8 erythromycin-resistant and erythromycin-resistant $S$. suis, respectively

\section{DISCUSSION}

Studies indicated that the number of erythromycinresistant S. suis strains is growing significantly and the resistant mechanisms are mainly due to Mef efflux (encoded by mefA gene, $\mathrm{M}$ phenotype) and 23sRNA methylase (encoded by ermB gene, IR and CR phenotype) (19,31). Resistance to 14- and 15-membered macrolides commonly 
used in clinical practice for treatment Streptococcal infection could be theoretically overcome by employing 16-membered compounds (20). Tilmicosin is a new macrolide antibiotic synthetically derived from tylosin, which is specially used in veterinary medicine $(10,15)$. Tiamulin was evaluated with tilmicosin because it has similar antimicrobial mechanism with macrolide and is commonly used to treat infections in swine and poultry $(3,5,13,27)$. In this study, the MIC and MBC results in Table 1 showed that tilmicosin and tiamulin were not only more active against susceptible $\mathrm{S}$. suis strains than erythromycin, but also maintain their antibacterial activity particularly against erythromycin-resistant strains with $M$ phenotype, thus extending their therapeutic possibilities.

Determination of MICs and the pharmacokinetics of antibiotics have been major factors in deciding the dosage and dosing interval of these drugs. This means that their blood concentrations ideally reach levels that are comparable with or higher than their MICs. However, in the clinical situation, after each dose of a regime, the concentrations of antibiotics fluctuate and subinhibitory concentrations may often occur between doses. Subinhibitory concentrations of antibiotics are known to have different effects on bacteria (17). Now the PAE and PA SME have attracted a greet deal of interest in recent years. They are well-known parameters that are correlated with the MICs and pharmacokinetics to analyze the effect of various dosing schedules $(25,26)$.

Our results of PAEs and PA SMEs from this study usefully expand those already gathered by other investigators $(1,9,22)$. One particularly interesting finding in our experiments is that erythromycin-resistant strains with $M$ phenotype show no PAE and PA SMEs at the usual erythromycin concentration, whereas the PAEs and PA SMEs of tilmicosin and tiamulin can be measured and have a duration comparable to that observed in susceptible strains. Because the PAE and PASME of tilmicosin and tiamulin against $S$. suis have not been determined, we compared our results with that of other similar studies. Similar results on PAE of rokitamycin against $S$. pyogenes were also observed by Braga (1). Compared to PAE of telithromycin against $S$. pyogenes (0.4-2.7 h), S. aureus (0.3-2.4 h) and S. pneumoniae (1.5-3.8 h), tilmicosin and tiamulin against $S$. suis (0.73-5.75 $\mathrm{h}$ and 1.15-6.11 h, respectively) tended to be longer (14). The PAE of tilmicosin(3.61-4.26 h) at 1×MIC after 2-h exposure was longer than that of $P$. multocida $(0.83 \mathrm{~h})$ and shorter than that of tilmicosin against A. pleuropneumoniae $(6 \mathrm{~h})(9)$.

There are several mechanisms associated with the PAEs of macrolides including uptake, ribosome binding, efflux, which mainly depend on the time that macrolides are detached from the ribosome $(4,33)$. Hydrophobic characteristic seems to help macrolide molecules to accumulate in cells and increases their activity. The 16member macrolides are more hydrophobic than their 14- or 15-member counterparts. Also the cohesive long-lasting binding of tilmicosin to ribosomes makes the efflux less effective than that of erythromycin. Our findings confirmed that the PAE of tilmicosin on erythromycin-resistant S. suis (0.73-5.6h) is even longer than that of erythromycin on susceptible strains (0.53-4.5h). Though little is known about the molecular basis of PAE and PA SME of tiamulin, it indeed has a good activity on erythromycin-resistant $S$. suis. Much work about the PAE mechanism of tiamulin remained to be continued.

In addition, our experiment indicated that PA SMEs lasted longer than PAEs. This means PAEs are not sufficient to predict the effects of antibiotics in the in vivo situations. The PA SME maybe more clinically relevant compared to the PAEs during intermittent dosage regimens, since suparinhibitory concentrations will be followed by exposure to subinhibitory concentrations in vivo. The interaction between sub-MIC of antibiotics and immune system is probably of great importance for therapeutic success when antibiotics are administered at long intervals. However, the relevance of these observations to clinical situation remains to be further investigated, since other variables such as pharmacokinetics may significantly change the clinical outcome of PAE and PA SME. 
Wang, L. et al.

\section{CONCLUSION}

The presented results revealed that tilmicosin and tiamulin were characterized by a powerful antibacterial activities and prolonged PAE and PASME against multidrug resistant $S$. suis. These results indicate that tilmicosn and tiamulin might be administered in longer time intervals without losing effectiveness than those already applied, and the greater success will be achieved in dosing regimes in large scale producton of swine and poultry. The knowledge on the PAE and PASME of a particular antibiotic may avoid unnecessary administration, which in turn reduces the stress to animals. To the most important, emerging resistance to macrolides in Streptococcus suis is a very important point, so appropriate regimes to minimize the development of resistance is crucial. These hypotheses remaine to be proved in a clinical setting that a modified dosage scheme based on prolonged PAE and PASME.

\section{ACKNOWLEDGEMENTS}

We are grateful to Dr. Huochun Yao and Dr. Hongjie Fan for providing some $S$. suis isolates. We are also appreciated that Dr Orhan Sahin helps to revise the manuscript.

\section{REFERENCE}

1. Braga, P.C.; Culici, M.; Dal, S. (2004). The post-antibiotic effects of rokitamycin (a 16-membered ring macrolide) on susceptible and erythromycin resistant strains of Streptococcus pyogenes. Int $J$ Antimicrob Agents, 24:56-62.

2. Bryskier, A.; Agouridas, C.; Gase, J.C. (1993). Classification of macrolide antibiotics. In: Bryskier A.; Butzler J.P.; Neu H.C.; Tulkens P.M. (eds). Macrolides: Chemistry, Pharmacology and Clinical Use. Paris: Arnette-Blackwell.

3. Burch, D.; Young, S.; Watson, E. (2007). Treatment of histomonosis in turkeys with tiamulin. Vet Rec, 161(25):864.

4. Cao, Z.; Zhong, P.; Ruan, X.; Merta, P.; Capobianco, J.O.; Flamm, R.K.; Nilius, A.M. (2004). Ribosome affinity and the prolonged molecular postantibiotic effect of cethromycin (ABT-773) in Haemophilus influenzae. Int J Antimicrob Agents ,24:362-8.

5. 5.Chengappa, M.M.; Pace, L.W.; Williams, J.A.; Herren, C.H.; Ascher, S.E. (1990). Efficacy of tiamulin against experimentally induced Streptococcus suis type-2 infection in swine. J Am Vet Med Assoc, 197: 1467-1470.
6. Clinical and Laboratory Standards Institute. (2008). Performance Standards for Antimicrobial Disk and Dilution Susceptibility Tests for Bacteria Isolated from Animals. Approved Standard-Third Edition. CLSI Document M31-A3 (ISBN 1-56238-659-x). CLSI, 940 West Valley Road, Suite 1400, Wayne, PA19087-1898, USA.

7. Craig, W.A.; Gudmundsson, S. (1996). The postantibiotic effect. In: Lorian V., editor. Antibiotics in Laboratory Medicine. 4th ed. Baltimore, MD: The Williams \& Wilkins Co., 296-329.

8. Daikos, G.K. (1991). Continuous versus discontinuous antibiotic therapy: the role of the post-antibiotic effect and other factors. $J$ Antimicrob Chemother, 27:157-160.

9. Diarra, M.S.; Malouin, F.; Jacques, M. (1999). Postantibiotic and physiological effects of tilmicosin, tylosin, and apramycin at subminimal and suprainhibitory concentrations on some swine and bovine respiratory tract pathogens. Int J Antimicrob Agents, 12: 229237.

10. Fricke, J.A.; Clark, C.R.; Boison, J.O.; Chirino-Trejo, M.; Inglis, T.E.; Dowling P.M. (2008). Pharmacokinetics and tissue depletion of tilmicosin in turkeys. $J$ Vet Pharmacol Ther,31(6):591-594.

11. Hendriksen, R.S.; Mevius, D.J.; Schroeter, A.; Teale, C.; Jouy, E.; Butaye, P.; Franco, A.; Utinane, A.; Amado, A.; Moreno, M.; Greko, C.; Stärk, K.D.; Berghold, C.; Myllyniemi, A.L.; Hoszowski, A.; Sunde, M.; Aarestrup, F.M. (2008). Occurrence of antimicrobial resistance among bacterial pathogens and indicator bacteria in pigs in different European countries from year 2002 - 2004: the ARBAO-II study. Acta Vet Scand, 50:19.

12. Higgins, R.; Gottschalk, M. (2006). Streptococcal diseases. In: Diseases of swine. Straw B.E.; Zimmerman J.J.; D'Allaire S.; Taylor D.J, (eds). 9 th ed, Ames, IA: Blackwell Publishing, 769-83.

13. Islam, K.M.; Afrin, S.; Das, P.M.; Hassan, M.M.; Valks, M.; Klein, U.; Burch, D.G.; Kemppainen, B.W. (2008). Compatibility of a combination of tiamulin and chlortetracycline with salinomycin in feed during a pulsed medication program coadministration in broilers. Poult Sci, 87(12):2528-34.

14. Jacobs, M.R.; Bajaksouzian, S.; Appelbaum, P.C. (2003). Telithromycin postantibiotic and post-antibiotic sub-MIC effects for 10 Gram-positive cocci. J Antimicrob Chemother, 52:809-812.

15. Jordan F.T.; Horrocks, B.K. (1996). The minimum inhibitory concentration of tilmicosin and tylosin for mycoplasma gallisepticum and Mycoplasma synoviae and a comparison of their efficacy in the control of Mycoplasma gallisepticum infection in broiler chicks. Avian Dis, 40(2):326-34.

16. Kennedy, K.J.; Jadeer, A.A.; Ong, C.W.; Senanayake, S.N.; Collignon, P.J. (2008). Two cases of Streptococcus suis endocarditis in Australian piggery workers. Med J Aust, 189: 413.

17. Lorian, V.; Gemmell, C.G. (1991). Effects of low antibiotic concentrations on bacteria: effects on ultrastructure, virulence, and susceptibility to immunodefenses. In: Lorian V. (ed). Antibiotics in Laboratory Medicine, 3rd edn. Baltimore, MA: Williams and Wilkins. 
18. Lun, Z.R.; Wang, Q.P.; Chen, X.G.; Li, A.X.; Zhu, X.Q. (2007). Streptococcus suis: an emerging zoonotic pathogen. Lancet Infect Dis, 7:201-209.

19. Martel, A.; Baele, M.; Devriese, L.A.; Goossens, H.; Wisselink, H.J.; Decostere, A.; Haesebrouck, F. (2001). Prevalence and mechanism of resistance against macrolides and lincosamides in Streptococcus suis isolates. Vet Microbiol, 83:287-297.

20. Mazzariol, A.; Koncan, R.; Vitali, L.A.; Cornaglia, G. (2007). Activities of 16-membered ring macrolides and telithromycin against different genotypes of erythromycin susceptible and erythromycinresistant Streptococcus pyogenes and Streptococcus pneumoniae. J Antimicrob Chemothe,59:1171-6.

21. 21.Ni, Y.X.; He, K.W.; Wang, J.C. (2002). Rapid detection of Streptococcus suis Type 2 by PCR. Chin J Vet Sci, 22: 474-476.

22. 22.Odenholt-Tornqvist, I.; Lowdin, E.; Cars, O. (1992). Postantibiotic sub-MIC effects of vancomycin, roxithromycin, sprafloxacin, and amikacin. Antimicrob Agents Chemother, 36: 1852-1858.

23. Odelholt-Tornqvist, I. (1993). Studies of the post-antibiotic effect and the post-antibiotic sub-MIC effect of meropenem. J Antimicrob Chemother, 31:881-892.

24. Pamela, R.T.; Charles, H.N.; David, P.N. (2000). Postantibiotic effect of trovafloxacin against Streptococcus pneumoniae, Haemophilus influenzae, and Neisseria meningitides in cerebrospinal fluid and broth culture media. Diagn Microbiol Infect Dis, 36:241-247.

25. Pankuch G.A.; Appelbaum P.C. (2006). Postantibiotic effect of ceftobiprole against 12 gram-positive organisms. Antimicrob Agents Chemother, 50:3956-3958.

26. 26.Plachouras, D.; Giamarellos-Bourboulis, E.J.; Kentepozidis, N.; Baziaka, F.; Karagianni, V.; Giamarellou, H. (2007). In vitro postantibiotic effect of colistin on multidrug-resistant Acinetobacter baumannii. Diagn Microbiol Infect Dis, 57:419-422.

27. Schuhmacher, A.; Bafundo, K.W.; Islam, K.M. ;. Aupperle, H.; Glaser, R.; Schoon, H.A.; Gropp, J.M. (2006). Tiamulin and semduramicin: effects of simultaneous administration on per formance and health of growing broiler chickens. Poult Sci, 85:441-5.

28. Seppälä, H.; Nissinan, A.; Yu, Q.; Huovinen, P. (1993). Three different phenotypes of erythromycin-resistant Streptococcus pyogenes in Finland. J Antimicrob Chemother, 32: 885-891.

29. Sutcliffe, J.; Grebe, T.; Tait-Kamradt, A.; Wondrack, L. (1996). Detection of erythromycin resistant determinants by PCR. Antimicrob Agents Chemother, 40: 2562-2566.

30. Vela, A.I. ; Moreno, M.A.; Cebolla, J.A.; González, S.; Latre, M.V.; Domínguez, L.; Fernández-Garayzábal, J.F. (2005). Antimicrobial susceptibility of clinical Strains of Streptococcus suis isolated from pigs in Spain. Vet Microbiol, 105: 143-147.

31. Wang, L.P.; Lu, C.P.; Tang, J.Q. (2004). Trends and phenotypes of resistance to macrolides in Streptococcus suis. Nanjing Nong Ye Da Хие Хие Вао, 4: 81-84.

32. Wisselink, H.J.; Veldman, K.T.; Van den Eede, C.; Salmon, S.A.; Mevius, D.J. (2006). Quantitative susceptibility of Streptococcus suis strains isolated from diseased pigs in Seven European countries to antimicrobial agents licensed in veterinary medicine. Vet Microbiol, 113:73-82.

33. Yano, H.; Kobayashi, T.; Okitsu, N.; Aoki, A.; Toriya, M.; Nakada, Y.; Sagai, H.; Iyobe, S.; Inoue, M. (2007). Comparison of antimicrobial and bactericidal activities and postantibiotic effects of macrolides antibiotics against clinical isolates, and examination of shape alteration by scanning electron microscope. Jpn J Antibiot, 60:31-46.

34. Yao, H.C.; Chen, G.Q.; Lu, C.P. (1999). Identification of swine Streptococcus isolates in Jiangsu Province during 1998. Nanjing Nong Yе Dа Хие Хие Baо, 22: 67-70.

35. Yu, H.J.; Jing, H.Q.; Chen, Z.H.; Xu, J.G.; Chen, Z.H.; Zhu, X.P.; Wang, H.; Liu, X.C.; Wang, S.W.; Liu, L.G.; Zu, R.Q.; Luo, L.Z.; Xiang, N.J.; Liu, H.L.; Zhong, W.J.; Liu, L.; Meng, L.; Yuan, H.; Gao, Y.J.; DU, H.M.; Ou, Y.B.; Ye, C.Y.; Jin, D.; Lv, Q.; Cui, Z.G.; Huang, Y.; Zhang, S.Y.; An, X.D.; Huang, T.; Zhou, X.Y.; Feng, L.; Pang, Q.D.; Shu, Y.L.; Wang, Y. (2006). Human Streptococcus suis outbreak, Sichuan, China. Emerg Infect Dis, 12:914-920.

36. Zhang, C.P.; Ning, Y.B.; Zhang, Z.Q.; Song, L.; Qiu, H.; Gao, H. (2008). In vitro antimicrobial susceptbility of Streptococcus suis strains isolated from clinically healthy sows in China. Vet Microbiol, 131: 386392. 